\title{
Differential expression of cocaine- and amphetamine-regulated transcript-immunoreactivity in the rat spinal preganglionic nuclei
}

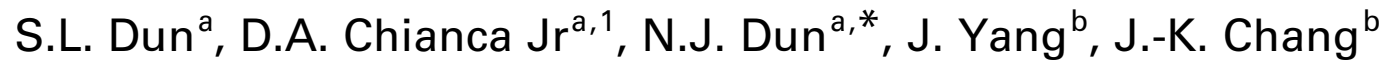 \\ ${ }^{a}$ Department of Pharmacology, James H. Quillen College of Medicine, East Tennessee State University, P.O. Box 70577, \\ Johnson City, TN 37614, USA \\ ${ }^{b}$ Phoenix Pharmaceuticals Inc., Belmont, CA 94043 USA
}

Received 7 August 2000; received in revised form 25 September 2000; accepted 26 September 2000

\begin{abstract}
The distribution of cocaine- and amphetamine-regulated transcript-like immunoreactivity (CART-LI) was investigated in the rat spinal cords with the use of an antiserum against the CART peptide fragment 55-102. CART-LI fibers were concentrated in the superficial layers of the dorsal horn of all segments. In addition to CART-LI fibers, intensely labeled somata were detected in the intermediolateral cell column (IML) and other sympathetic preganglionic nuclei of the thoracolumbar segments. In the lumbosacral segments, CART-LI fibers but not somata were seen in the sacral parasympathetic nucleus. Double-labeling the spinal sections with choline acetyltransferase (ChAT)-antisera and CARTantisera revealed that the large majority of ChAT-positive somata in the sympathetic preganglionic nuclei were CARTpositive, whereas ChAT-positive somata in the parasympathetic preganglionic nuclei were CART-negative. Our results show that CART-LI is selectively expressed in a population of sympathetic preganglionic neurons (SPNs), but not in parasympathetic preganglionic neurons (PPNs) of the rat. (c) 2000 Elsevier Science Ireland Ltd. All rights reserved.
\end{abstract}

Keywords: Intermediolateral cell column; Parasympathetic preganglionic neurons; Sympathetic preganglionic neurons; Spinal cord

Cocaine- and amphetamine-regulated transcript (CART) was first identified in the rat striatum where the levels were up-regulated following acute administration of cocaine and amphetamine [2]. Immunohistochemical and in situ hybridization studies have since revealed an extensive distribution of CART-like immunoreactivity (LI) and mRNA in the rat brain, pituitary and adrenal medulla [1,6-8]. Western blot analysis of rat tissues indicates that there are at least six different CART peptides whose molecular weights vary from 4-14 $\mathrm{kDa}$ [9]. The higher molecular weight peptides are thought to be preproCART and proCART; whereas, the lower molecular weight peptides $\mathrm{CART}_{55-102}$ and $\mathrm{CART}_{62-102}$ may be the physiologically active fragments $[8,9]$.

In the rat spinal cord, CART-LI is concentrated in networks of fibers in lamina I and II of the dorsal horn, and in neurons of the intermediolateral cell column (IML) of the thoracolumbar segments [5,7]. However, the distribution of CART-LI in the parasympathetic preganglionic nuclei of the lumbosacral segment has yet to be investi-

\footnotetext{
* Corresponding author. Fax: +1-423-439-8773.

E-mail address: dunnae@etsu.edu (N.J. Dun).

${ }^{1}$ Department of Biological Sciences, Federal University of Ouro Preto, Ouro Preto, MG 35400-000, Brazil
}

gated. The present study was undertaken to address this issue.

Adult male and female Sprague-Dawley rats, weighing 200-250 g, from Harlan (Indianapolis, IN) were used in these studies. Experimental protocols were approved by the University Committee on Animal Care.

Rats were anesthetized with urethane $(1.2 \mathrm{~g} / \mathrm{kg}$, i.p.) and perfused intracardially with chilled $0.1 \mathrm{M}$ phosphatebuffered saline (PBS) followed by freshly prepared $4 \%$ paraformaldehyde in PBS. Spinal cords were removed, postfixed in the same fixative for $2 \mathrm{~h}$, and stored in $30 \%$ sucrose/ PBS solution overnight. Transverse or horizontal $40 \mu \mathrm{m}$ spinal cord sections were prepared by a Vibratome and processed for CART-LI using the avidin-biotin complex method as described previously $[3,4]$. Sections were first treated with $3 \% \mathrm{H}_{2} \mathrm{O}_{2}$ to quench endogenous peroxidase, washed and blocked with $10 \%$ normal goat sera, and incubated with CART-antisera (10 000-16 000 dilution with $0.4 \%$ Triton X-100 and $1 \%$ bovine serum albumin in PBS; Phoenix Pharmaceuticals, Inc.). The CART-antiserum, a rabbit polyclonal directed against the peptide fragment 55-102, exhibits $100 \%$ cross-reactivity with the CART peptide (Phoenix Pharmaceuticals, Inc.). Two sets of control 
experiments were performed. First, the primary antisera were omitted from the staining procedures. Second, sections were processed with CART-antisera pre-absorbed with the CART peptide ${ }_{55-102}(10 \mu \mathrm{g} / \mathrm{ml}$, Phoenix Pharmaceuticals, Inc.) overnight. Several thoracolumbar and lumbosacral sections from each rat were set aside for double-labeling experiments in which sections were incubated sequentially with choline acetyltransferase (ChAT)-antisera and CARTantisera from different hosts, as described previously [3]. Goat polyclonal ChAT-antisera were from Chemicon International, Inc. (Temecula, CA). Sections were first processed for ChAT-LI by incubating in ChAT-antisera (1:300 with $0.4 \%$ Triton X-100), later in biotin and then avidin fluorescein $\mathrm{D}$. The second step involved the incubation of tissue sections in CART-antisera (1:1000 with $0.4 \%$ Triton X100), biotin and avidin Texas Red.

The spinal cords from nine rats were processed for CART-LI. Approximately 20 transverse sections of cervical, thoracic, lumbar and sacral spinal segments from six rats and several horizontal sections from three rats were examined. The pattern of distribution of CART-LI in the spinal cord of male $(n=6)$ and female $(n=3)$ rats was similar. A feature common to all the segments was the presence of fairly dense networks of CART-LI fibers in laminae I and II of the dorsal horn; the deeper laminae and ventral horn exhibited much fewer CART-LI fibers (Fig. 1A,C). A few labeled somata of small diameter $(<10 \mu \mathrm{m})$ were scattered throughout the dorsal horn.
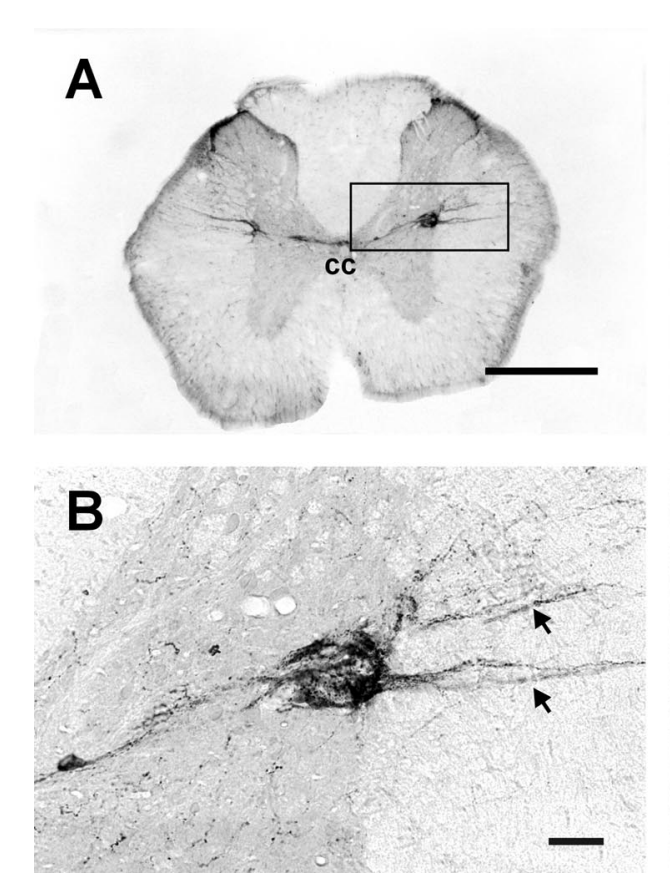

CART-LI somata were conspicuously present in the IML of thoracolumbar segments (Figs. 1A,B, 2A,B and 3A,B). Labeled somata were also seen in other autonomic nuclei including the intermediomedial nucleus and central autonomic nucleus (Figs. 1-3). In the transverse sections, CART-LI cell processes extended medially toward the central canal, as described for rat sympathetic preganglionic neurons (SPNs) intracellularly labeled with lucifer yellow [11]. In the horizontal sections, labeled SPNs and cell processes were arranged in a ladder-like network between the lateral horn and central canal (Fig. 2).

With respect to the rat lumbosacral cord, CART-LI somata were not detected in the parasympathetic preganglionic nuclei (Figs. 1C,D, 2C,D and 3C,D). Instead, CART-LI fibers were present in the parasympathetic preganglionic nuclei and around the central canal (Figs. 1C and 2D). The lack of CART-LI somata in the parasympathetic preganglionic nuclei was clearly evident in the horizontal sections (Fig. 2C,D).

In control sections processed with CART-antisera preabsorbed with the peptide overnight, immunoreactivity was not seen in any of the spinal sections (not shown, see Ref. [3]).

In the case of thoracolumbar sections double-labeled with ChAT- and CART-antisera, the large majority of ChAT-LI somata in the IML of thoracolumbar segments were CARTpositive and vice versa. A few ChAT-LI somata were CART-negative, whereas some CART-positive neurons
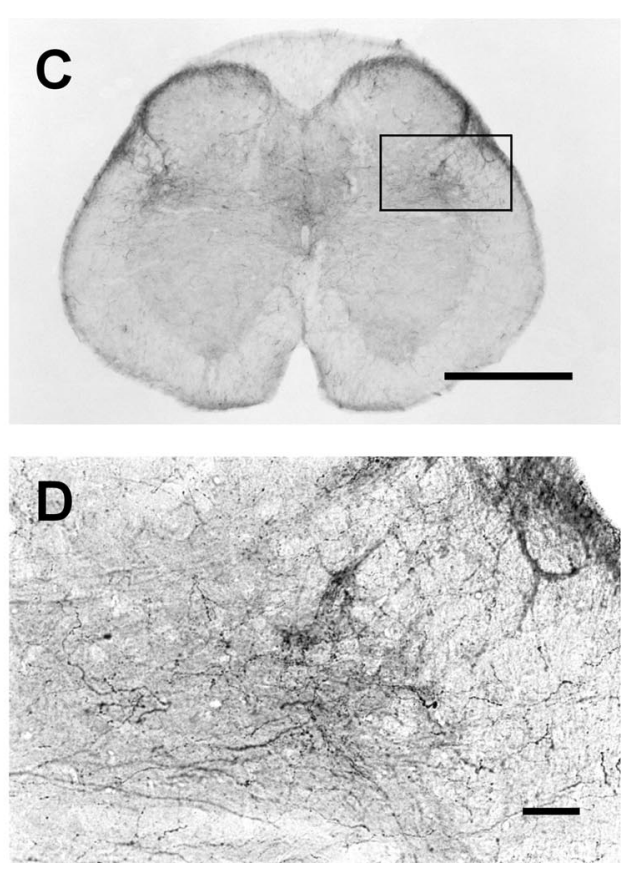

Fig. 1. Photomicrographs of transverse sections through the rat thoracic and lumbosacral spinal cord labeled with CART-antisera. (A) Low magnification of a thoracic segment where CART-LI fibers are concentrated in laminae I and II, and CART-LI somata and cell processes are seen in the IML, intermediomedial nucleus and central autonomic nucleus. (B) Higher magnification of an area outlined in (A) where CART-LI somata and fibers in the IML are clearly seen; several CART-positive fibers (arrows) appear to project into the IML area from the lateral funiculus. (C) Low magnification of a lumbosacral segment where CART-LI fibers are concentrated in laminae I and II and lateral gray area. (D) Higher magnification of an area outlined in (C) where numerous CART-LI fibers, but not somata, are seen in the lateral gray area. Scale bar: $250 \mu \mathrm{m}$ for $(A, C) ; 50 \mu \mathrm{m}$ for $(B, D)$. 

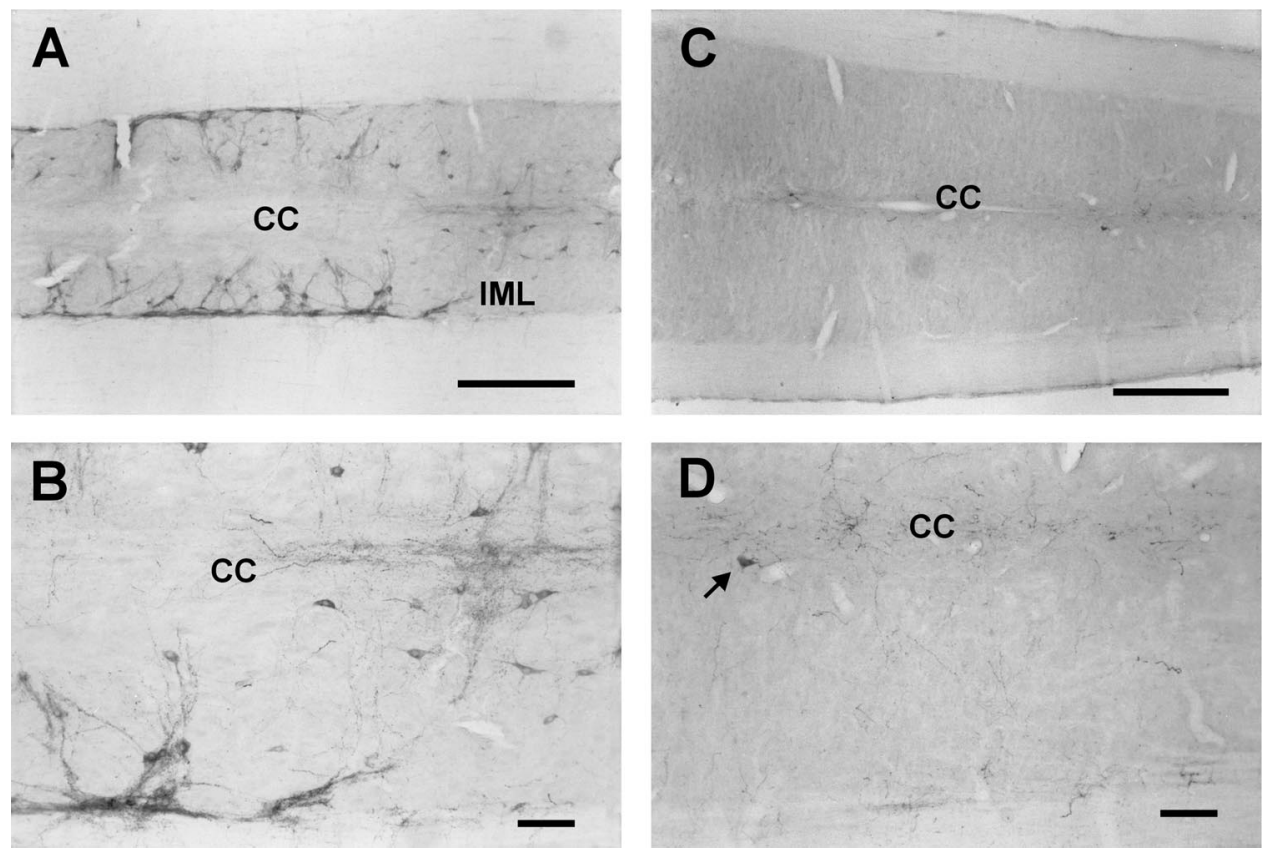

Fig. 2. Photomicrographs of horizontal sections through thoracic and lumbosacral spinal cord labeled with CART-antisera. (A) Low magnification of a horizontal thoracic segment showing the distribution of CART-immunoreactive neurons and cell processes arranged in a ladder-like network. (B) Higher magnification of (A) showing CART-immunoreactive cells in the IML and lateral to the central canal (CC). (C) Low magnification of a horizontal lumbosacral segment showing a nearly total absence of CART-LI somata. (D) Higher magnification of $(C)$ showing a solitary CART-LI cell (arrow) lateral to the central canal; CART-LI fibers are seen around the central canal and along the lateral gray. Scale bar: $250 \mu \mathrm{m}$ for $(A, C) ; 100 \mu \mathrm{m}$ for $(B, D)$.
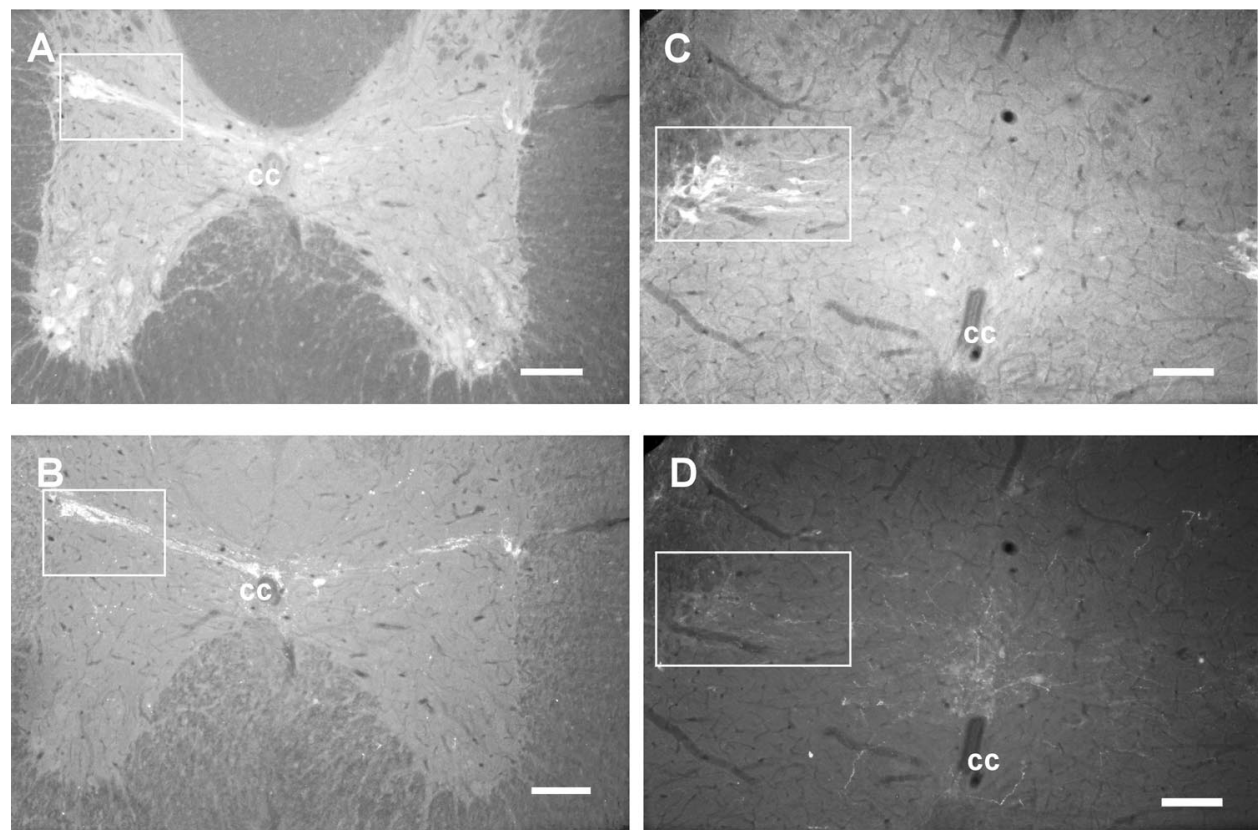

Fig. 3. Photomicrographs of transverse sections through thoracic and lumbosacral spinal cord double-labeled with ChAT- and CARTantisera. $(A, C)$ are labeled with ChAT-antisera, and (B,D) are labeled with CART-antisera. (A) ChAT-LI neurons are seen in the IML, which is outlined, and around the central canal; immunoreactive cell processes extend medially toward the central canal. ChAT-positive cells are also noted in the ventral horn. (B) same section as (A); CART-immunoreactive cells in the IML, which is outlined, and around the central canal, some of which overlap with ChAT-positive cells shown in (A). CART-LI cells are absent in the ventral horn. (C) ChAT-LI cells are seen in the parasympathetic preganglionic nucleus, which is outlined, and around the central canal. (D) same section as (C); CART-LI cells are not seen in the parasympathetic preganglionic nucleus, which is outlined; several positively labeled cells are noted dorsal to the central canal. Scale bar: $100 \mu \mathrm{m}$. 
were ChAT-negative (Fig. 3A,B). Ventral horn ChAT-LI neurons were CART-negative (Fig. 3A,B). There was no evidence of CART-LI in ChAT-positive neurons in the parasympathetic preganglionic nuclei of lumbosacral sections from any of the rats (Fig. 3C,D).

The present study confirms and extends the earlier observations relative to the distribution of CART-LI in the rat spinal cord [5,7]. The CART-antisera used in our study were directed against the CART peptide ${ }_{55-102}$, whereas the CART-antisera used in an earlier study were raised against

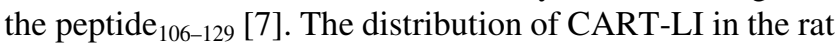
brain and spinal cord as revealed by these two different antisera was similar [5,7]. Further, CART-LI was not detected in spinal sections incubated in CART-antisera pre-absorbed with the peptide, attesting to the specificity of the antisera used in our study.

As noted in the earlier study [5,7], CART-LI fibers were present throughout the gray matter, with the highest concentration in the superficial layers of the dorsal horn. Insofar as the somata are concerned, a few smaller diameter CART-LI neurons were scattered in the dorsal horn. The biological action of CART on dorsal horn neurons remains to be fully explored. Initial studies showed that an intrathecal administration of CART decreases hindpaw withdrawal latency in the mice, suggesting that the peptide may sensitize dorsal horn neurons to thermal stimuli [10].

The most conspicuous group of CART-LI cell bodies in the spinal cord is found in the lateral horn of the thoracolumbar cord. On the basis of their location, morphology and co-expression of ChAT-LI, the CART-LI neurons in the IML are presumed to be SPNs. On the other hand, CARTLI was not detected in ChAT-positive neurons of the parasympathetic preganglionic nuclei of the lumbosacral cord. Interestingly, ChAT-positive ventral horn neurons were CART-negative as well. The lack of expression of CARTLI in parasympathetic preganglionic neurons (PPNs) and ventral horn neurons, as revealed by our antisera, is probably not due to the presence of a different form of CART peptide, because CART mRNA is reported to be absent in the rat lumbar and sacral cord by in situ hybridization [1].

Projecting to different peripheral target ganglion cells notwithstanding, SPNs in the thoracolumbar and PPNs in the lumbosacral cord are not known to differ with respect to their transmitter phenotypes. Both types of neurons are cholinergic and their axons release acetylcholine as the principal transmitter. Viewed in this context, our results provide the first evidence that CART peptide is selectively expressed in SPNs but not in PPNs.

This study was supported by NIH Grants NS18710 and NS39646 from the Department of Health and Human Services.

[1] Couceyro, P.R., Koylu, E.O. and Kuhar, M.J., Further studies on the anatomical distribution of CART by in situ hybridization, J. Chem. Neuroanat., 12 (1997) 229-241.

[2] Douglass, J., McKinzie, A.A. and Couceyro, P., PCR differential display identifies a rat brain mRNA that is transcriptionally regulated by cocaine and amphetamine, $\mathrm{J}$. Neurosci., 15 (1995) 2471-2481.

[3] Dun, N.J., Dun, S.L., Wu, S.Y., Forstermann, U., Schmidt, H.H.H.W. and Tseng, L.F., Nitric oxide synthase immunoreactivity in the rat, mouse, cat and squirrel monkey spinal cord, Neuroscience, 54 (1993) 843-857.

[4] Dun, N.J., Miyazaki, T., Tang, H. and Dun, E.C., Pituitary adenylate cyclase activating polypeptide immunoreactivity in the rat spinal cord and medulla: implication of sensory and autonomic function, Neuroscience, 73 (1996) 677-686.

[5] Dun, N.J., Dun, S.L., Kwok, E.H., Yang, J. and Chang, J.-K., Cocaine- and amphetamine-regulated transcript-immunoreactivity in the rat sympatho-adrenal axis, Neurosci. Lett., 283 (2000) 97-100.

[6] Koylu, E.O., Couceyro, P.R., Lambert, P.D., Ling, N.C., DeSouza, E.B. and Kuhar, M.J., Immunohistochemical localization of novel CART peptides in rat hypothalamus, pituitary and adrenal gland, J. Neuroendocrinol., 9 (1997) 823-833.

[7] Koylu, E.O., Couceyro, P.R., Lambert, P.D. and Kuhar, M.J., Cocaine- and amphetamine-regulated transcript peptide immunohistochemical localization in the rat brain, J. Comp. Neurol., 391 (1998) 115-132.

[8] Kuhar, M.J. and Dall Vechia, S.E., CART peptides: novel addiction- and feeding-related neuropeptides, Trends Neurosci., 22 (1999) 316-320.

[9] Kuhar, M.J. and Yoho, L.L., CART peptide analysis by Western blotting, Synapse, 33 (1999) 163-171.

[10] Ohsawa, M., Dun, S.L., Tseng, L.F., Chang, J.-K. and Dun, N.J., Decrease of hindpaw withdrawal latency by cocaineand amphetamine-regulated transcript peptide to the mouse spinal cord, Eur. J. Pharmacol., 399 (2000) 165-169.

[11] Shen, E. and Dun, N.J., Neonatal rat sympathetic preganglionic neurons intracellularly labelled with lucifer yellow in thin spinal cord slices, J. Auton. Nerv. Syst., 29 (1990) 247254. 